\title{
Distance calculation between photovoltaic arrays fixed on sloping ground
}

\author{
Xiu-Shui Ma $^{\mathrm{a}, *}$, Guang-Hui Yao ${ }^{\mathrm{a}, \mathrm{b}, \mathrm{c}}$, Ling-Jian Ye ${ }^{\mathrm{a}}$, Xiong-Fei Zhi ${ }^{\mathrm{a}}$ and Shu-Ming Zhang ${ }^{\mathrm{a}}$ \\ ${ }^{a}$ Ningbo Institute of Technology, School of Information Science and Engineering, Zhejiang University, \\ Ningbo, Zhejiang, China \\ ${ }^{\mathrm{b}}$ Department of Control Science and Engineering, Zhejiang University, Hangzhou, Zhejiang, China \\ ${ }^{\mathrm{c}}$ The IT Electronics Eleventh Design \& Research Institute Scientific and Technological Engineering \\ Corporation Limited, Hangzhou Branch, Hangzhou, Zhejiang, China
}

\begin{abstract}
The distance calculation between two photovoltaic arrays is important in the design of grid-connected and grid-off power generation. It is easy to calculate the distance between two photovoltaic arrays on horizontal ground, but on the sloping ground existed in practical projects, it is more complicated. This paper introduces a kind of analytical geometry method to solve the problem of distance calculation between two photovoltaic arrays fixed on sloping ground.
\end{abstract}

Keywords: Solar power generation, photovoltaic array distance, sloping ground, projected length

\section{Introduction}

Solar energy is a clean and efficient energy, and solar energy power station has been widely used. Solar power generation is a low density of energy. Large scale solar grid-connected station takes a lot of land resources. The reference [1/2] introduce the influence of the distance between two photovoltaic arrays and the power generation capacity in per unit area. If the distance of the photovoltaic arrays is too large, the power generation capacity in per unit area will be reduced, therefore this phenomenon cause a serious waste of land resources, especially in the area the land resources are rare. But if the distance of the photovoltaic arrays is too small, the time of the effective power generation will be reduced because of the photovoltaic arrays' shadow in front shading the back row earlier, and the power generation capacity is also affected. Therefore, it is greatly significant for saving land resources, reducing the cost and increasing power generation capacity to design the distance between the photovoltaic arrays properly.

It is easy to calculate the distance between two photovoltaic arrays on horizontal ground of the south. The reference [3] introduces the calculation method about the distance between two photovoltaic arrays on horizontal ground of the south. The reference [4] introduces the calculation method about the distance between two photovoltaic arrays on northern slope area. The reference [5] studies the variety of shadow and the optimum distribution of arrays tracking system. But in general, the calculation methods about the distance between two photovoltaic arrays have not been founded in relevant design specifications or references. The conditions on non-horizontal ground are often appeared in practical engineering,

\footnotetext{
${ }^{*}$ Corresponding author: Xiu-Shui Ma, Ningbo Institute of Technology, School of Information Science and Engineering, Zhejiang University, Ningbo, Zhejiang, China. Tel.: +86 1368589 1614; Fax: +86 0574 88229505; E-mail: mxsh63@aliyun.com. 


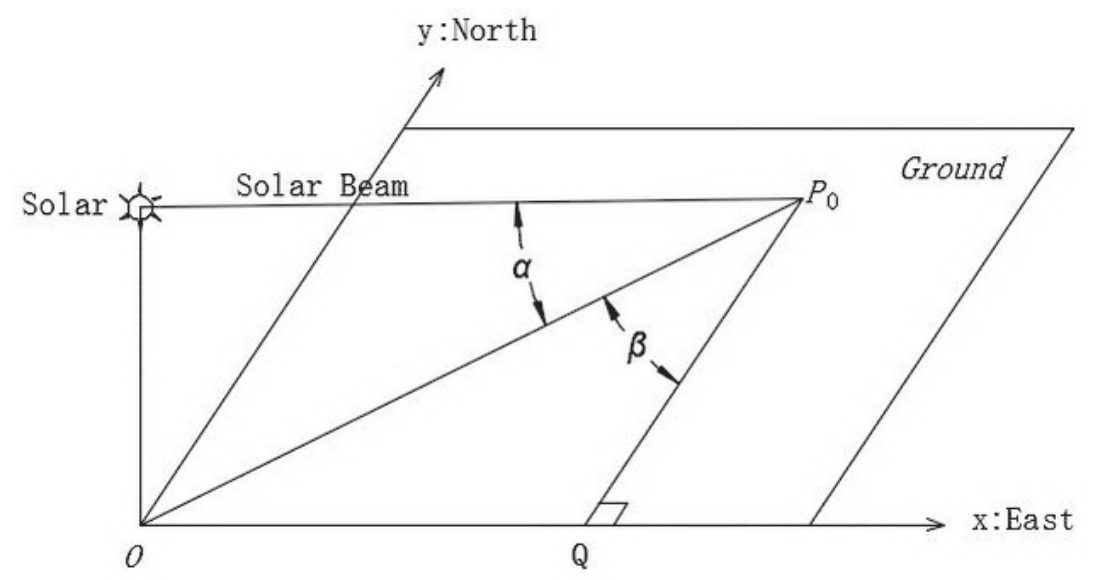

Fig. 1. Solar altitude angle and azimuth angle.

for example, mountain slope, the lake dam and so on. Consequently, this paper studies a kind of general calculation method to solve the problems about the distance calculation between two photovoltaic arrays fixed on sloping ground.

\section{The calculation of the distance between two photovoltaic arrays on horizontal ground}

The calculation of the distance between two photovoltaic arrays on horizontal ground can be calculated by solar altitude angle and azimuth angle [6-8].

Altitude angle $\alpha$ is:

$$
\sin \alpha=\sin \varphi \sin \delta+\cos \varphi \cos \delta \cos \omega
$$

Azimuth angle $\beta$ is:

$$
\begin{aligned}
& \sin \beta=\frac{\cos \delta \sin \omega}{\cos \alpha} \\
& \cos \beta=\frac{\sin \alpha \sin \varphi-\sin \delta}{\cos \alpha \cos \varphi}
\end{aligned}
$$

where, $\varphi$ is local latitude, $\delta$ is solar declination angle, $\omega$ is solar hour angle. The solar altitude angle and azimuth angle are shown in Fig. 1. Plane xoy is the ground, y points to North and x points to East. $P_{0}$ is a point of solar beam and ground. $P_{0} Q$ points to the south. The angle between solar beam and $O P_{0}$ is the altitude angle $\alpha$ and the angle between $Q P_{0}$ and $O P_{0}$ is the azimuth angle $\beta$.

The design specifications in photovoltaic power station require the distance between two photovoltaic arrays to satisfy the following conditions: the photovoltaic arrays are not shielded each other in the local solar time from 9:00 to 15:00, in other words, the photovoltaic arrays are not shielded each other in the Winter Solstice of the Northern Hemisphere [9]. In the Winter Solstice, the solar declination angle is $-23^{\circ} 27^{\prime}$, and the solar hour angle is $-45^{\circ}$ at 9:00 in the morning and $+45^{\circ}$ at 3:00 in the afternoon.

The calculation about the shadow length of photovoltaic arrays on horizontal ground of the south is showed as follows through the solar altitude angle $\alpha$ and azimuth angle $\beta$. 
Shadow length $D_{1}$ in north-south direction:

$$
D_{1}=H \frac{\cos \beta}{\tan \alpha}=H \frac{\tan \varphi+0.61345}{1-0.61345 \tan \varphi}
$$

Shadow length $D_{2}$ in east-west direction:

$$
D_{2}=H \frac{\sin \beta}{\tan \alpha}=\frac{H}{0.61345 \sin \varphi-\cos \varphi}
$$

In the Eqs (4) and (5), $H$ is the height of the photovoltaic arrays vertex from the ground.

When the photovoltaic arrays have an azimuth angle $\gamma$ with regard to the south direction, the solar azimuth angle $\beta$ will be changed into $\beta-\gamma$ in the Eqs (4) and (5). In general, the azimuth angle should be less than $20^{\circ}$ when non-horizontal installed, because the transfer efficiency of photovoltaic arrays is seriously affected by the bigger azimuth angle.

Considering the factor of photovoltaic site's terrain height difference, the distance between two photovoltaic arrays is generally designed longer than the shadow length in north-south direction.

\section{The calculation of the distance between two photovoltaic arrays on sloping ground in north-south direction}

The solar photovoltaic arrays on sloping ground in north-south direction are generally arranged in south direction. As shown in Fig. 2, $\mathrm{OBCD}$ stands for horizontal ground and $\mathrm{OB}^{\prime} \mathrm{C}^{\prime} \mathrm{D}^{\prime}$ stands for sloping ground. The north direction of sloping ground $\mathrm{OB}^{\prime} \mathrm{C}^{\prime} \mathrm{D} /$ is high while the south direction is low, the dip angle of sloping ground $\mathrm{OB}^{\prime} \mathrm{C}^{\prime} \mathrm{D}^{\prime}$ with the horizontal ground $\mathrm{OBCD}$ is $\theta$. Highest point of the photovoltaic arrays is point $\mathrm{A}$. AC is solar beam that passed point $\mathrm{A}$ and arrived the horizontal ground. The height OA of the photovoltaic arrays' vertex from the ground assumed as $H$, and $H$ can be calculated by dip angle and the length of the photovoltaic arrays. At 3:00 in the afternoon, $\mathrm{OC}^{\prime}$ and $\mathrm{OC}$ is the shadow of $\mathrm{OA}$ on the sloping ground and the horizontal ground. The vertical line of $\mathrm{OD}^{\prime}$ is AE.

The shadow length of the horizontal ground can be calculated by the Eqs (1) to (5). That is $\mathrm{OD}=D_{1}$, $\mathrm{OB}=D_{2}$. Now the distance between the photovoltaic arrays from the front to back in sloping ground is $\mathrm{OD}^{\prime}$. The results can be got from triangle area equation $\Delta \mathrm{ODA}=\Delta \mathrm{OD}^{\prime} \mathrm{A}+\Delta \mathrm{OD}^{\prime} \mathrm{D}$ :

$$
\frac{1}{2} \mathrm{OD} \times \mathrm{OA}=\frac{1}{2} \mathrm{OD}^{\prime} \times \mathrm{AE}+\frac{1}{2} \mathrm{OD}^{\prime} \times \mathrm{OD} \times \sin \theta
$$

Substitute $\mathrm{OD}=\mathrm{D}_{1}, \mathrm{OA}=\mathrm{H}, \mathrm{AE}=\mathrm{OA} \cos \theta=\mathrm{H} \cos \theta$ into the equation above and the minimum distance between photovoltaic arrays from the front to back on sloping ground is:

$$
\mathrm{OD}^{\prime}=\frac{D_{1} H}{H \cos \theta+D_{1} \sin \theta}
$$

Similarly, the projection of the photovoltaic arrays' shadow $\mathrm{OC}^{\prime}$ on sloping ground in east-west direction $\mathrm{OB}^{\prime}$ is:

$$
\mathrm{OB}^{\prime}=\frac{D_{2} H \cos \theta}{H \cos \theta+D_{1} \sin \theta}
$$

If the north of the sloping ground is low and the south is high, the Eqs (6) and (7) can also be used, but the dip angle $\theta$ is negative. 


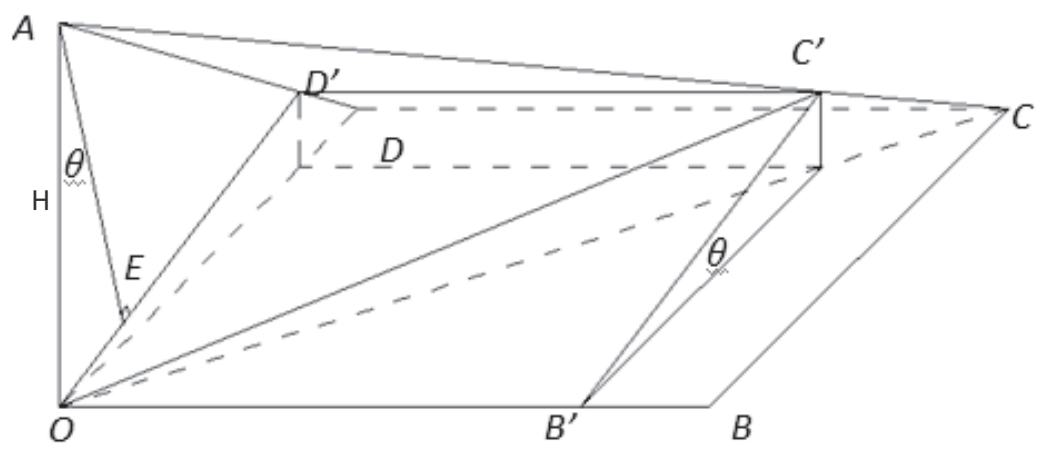

Fig. 2. Photovoltaic arrays' projection of the sloping ground in north-south direction.

\section{The calculation of the distance between two photovoltaic arrays on sloping ground in east-west direction}

The solar photovoltaic arrays on sloping ground in east-west direction are generally arranged in south direction as well. As shown in Fig. 3, the east direction of the sloping ground $\mathrm{OB}^{\prime} \mathrm{C}^{\prime} \mathrm{D}^{\prime}$ is high while the west direction is low, the dip angle of the sloping ground $\mathrm{OB}^{\prime} \mathrm{C}^{\prime} \mathrm{D}^{\prime}$ with the horizontal ground $\mathrm{OBCD}$ is $\theta$. The vertex of the photovoltaic arrays is point A. Height OA of the photovoltaic arrays' vertex from the ground assumed as $H$, and $H$ can be calculated by the dip angle and the length of photovoltaic arrays. At 3:00 in the afternoon, $\mathrm{OC}^{\prime}$ and $\mathrm{OC}$ is the shadow of $\mathrm{OA}$ on the sloping ground and the horizontal ground. The photovoltaic arrays' shadow is projected on the uphill of the sloping ground. The vertical line of $\mathrm{OB}^{\prime}$ is $\mathrm{AE}$.

The shadow length of the horizontal ground can be calculated by the Eqs (4) and (5). That is OD = $D_{1}, \mathrm{OB}=D_{2}$. The results can be got from triangle area equation $\Delta \mathrm{OAB}=\Delta \mathrm{OB}^{\prime} \mathrm{A}+\Delta \mathrm{OB}^{\prime} \mathrm{B}$ :

$$
\frac{1}{2} \mathrm{OB} \times \mathrm{OA}=\frac{1}{2} \mathrm{OB}^{\prime} \times \mathrm{AE}+\frac{1}{2} \mathrm{OB}^{\prime} \times \mathrm{OB} \times \sin \theta
$$

Substitute $\mathrm{OB}=D_{2}, \mathrm{OA}=H, \mathrm{AE}=\mathrm{OA} \cos \theta=H \cos \theta$ into the equation above and the projection of the photovoltaic arrays' shadow $\mathrm{OC}^{\prime}$ on sloping ground in east-west direction $\mathrm{OB}^{\prime}$ is:

$$
\mathrm{OB}^{\prime}=\frac{D_{2} H}{H \cos \theta+D_{2} \sin \theta}
$$

The minimum distance between photovoltaic arrays from the front to back on sloping ground is:

$$
\mathrm{OD}^{\prime}=\frac{D_{1} H \cos \theta}{H \cos \theta+D_{2} \sin \theta}
$$

The conditions above are photovoltaic arrays projected on the uphill and the Eqs (8) and (9) are also applied to the conditions that projected on the downhill when dip angle $\theta$ is changed into negative.

\section{The calculation of the distance between two photovoltaic arrays on sloping ground in general}

The calculation of the distance between two photovoltaic arrays on sloping ground in north-south and east-west direction is introduced above. But the sloping ground along arbitrary direction is a kind of 


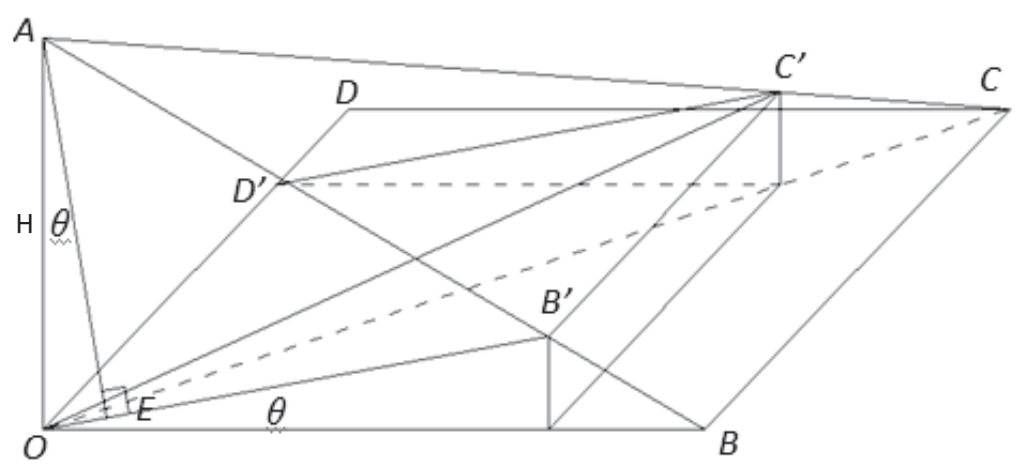

Fig. 3. Photovoltaic arrays' projection of the sloping ground in east-west direction.

more common condition. Supposing the dip angle of the sloping ground is $\gamma$ with the south direction, the arrangement direction of the photovoltaic arrays is also $\gamma$ with regard to the south. The solar azimuth angle $\beta$ will be changed into $\beta-\gamma$ in Eqs (4) and (5). Then the Eqs (4) and (5) can be transformed into the following:

$$
\begin{aligned}
& D_{1}^{\prime}=H \frac{\cos (\beta-\gamma)}{\tan \alpha}=D_{1} \cos \gamma+D_{2} \sin \gamma \\
& D_{2}^{\prime}=H \frac{\sin (\beta-\gamma)}{\tan \alpha}=D_{2} \cos \gamma-D_{1} \sin \gamma
\end{aligned}
$$

Substitute the Eqs (10) and (11) into Eqs (6) and (7). The minimum distance between photovoltaic arrays from the front to back on sloping ground is $D_{1}{ }^{\prime} H /\left(H \cos \theta+D_{1}{ }^{\prime} \sin \theta\right)$. The component in right and left direction about the photovoltaic arrays' shadow on sloping ground is $D_{2}{ }^{\prime} H \cos \theta /\left(H \cos \theta+D_{1}{ }^{\prime} \sin \theta\right)$. Among them, $H$ is the vertical distance between the photovoltaic arrays topside and sloping ground; $\theta$ is dip angle of the ground.

The method above is not applied if the photovoltaic arrays are not arranged along with the sloping ground direction. Considering arbitrary arrangement of the photovoltaic arrays in arbitrary sloping ground, a kind of analytical geometry method is introduced below [10].

Firstly, set up a three-dimensional Cartesian coordinate system, suppose a point on the ground as the origin of coordinate, the south direction as $\mathrm{x}$-axis, the east direction as $\mathrm{y}$-axis, the vertical upward as z-axis, As shown in Fig. 4. So an arbitrary point in three-dimensional space can be represented as $\boldsymbol{p}=$ $[x, y, z]^{T}$. Then the equation about the sloping ground will be set up. Supposing $\boldsymbol{n}$ is the normal of the ground, $\alpha_{1}$ is the attitude angle of the ground normal, i.e., the angle of the normal with its projection on horizontal ground, it is also dip angle of the sloping ground. $\beta_{1}$ is azimuth angle of the ground normal, i.e., the angle of ground normal's projection on horizontal ground with the south direction, south by east is positive, and south by west is negative.

Vector of the ground normal is:

$$
\boldsymbol{n}=R\left[z, \beta_{1}\right] R\left[y, \alpha_{1}\right]\left[\begin{array}{lll}
0 & 0 & 1
\end{array}\right]^{T}
$$

Where, $R\left[z, \beta_{1}\right]$ and $R\left[y, \alpha_{1}\right]$ is the spin matrix around $\mathrm{z}$-axis and $\mathrm{y}$-axis.

$$
R\left[z, \beta_{1}\right]=\left[\begin{array}{ccc}
\cos \beta_{1} & -\sin \beta_{1} & 0 \\
\sin \beta_{1} & \cos \beta_{1} & 0 \\
0 & 0 & 1
\end{array}\right]
$$




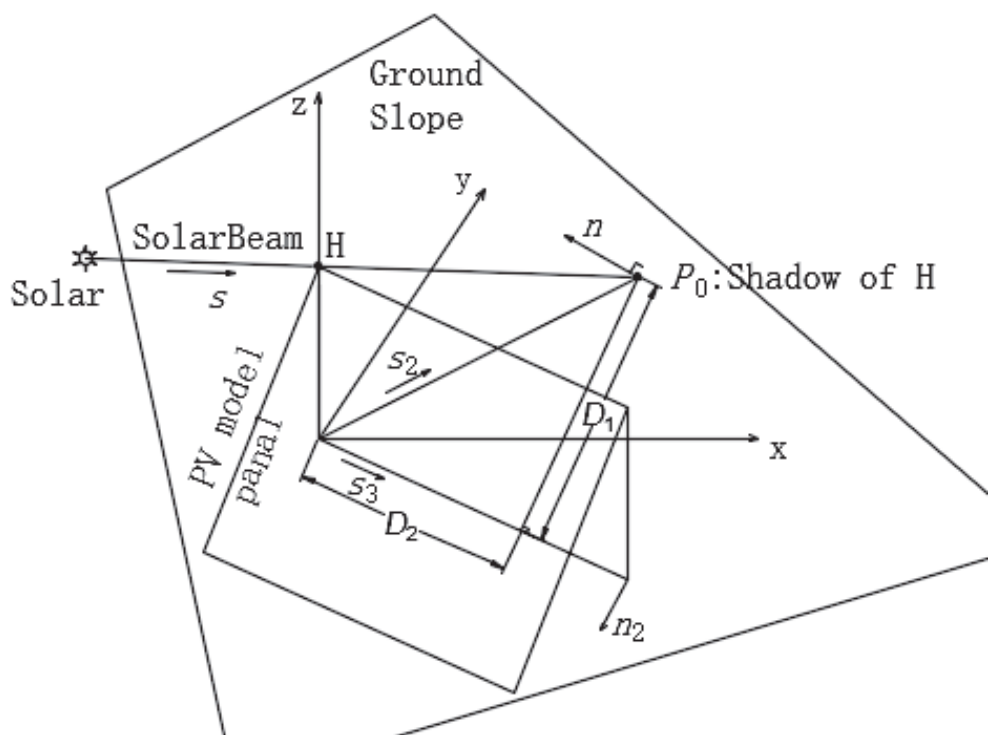

Fig. 4. Photovoltaic arrays' projection of the sloping ground in general condition.

$$
R\left[y, \alpha_{1}\right]=\left[\begin{array}{ccc}
\cos \alpha_{1} & 0 & \sin \alpha_{1} \\
0 & 1 & 0 \\
-\sin \alpha_{1} & 0 & \cos \alpha_{1}
\end{array}\right]
$$

Substitute $R\left[z, \beta_{1}\right]$ and $R\left[y, \alpha_{1}\right]$ into the Eq. (12), then

$$
\boldsymbol{n}=\left[\begin{array}{lll}
\cos \beta_{1} \sin \alpha_{1} & \sin \beta_{1} \sin \alpha_{1} & \cos \alpha_{1}
\end{array}\right]^{T}
$$

Equation of the sloping ground through the origin point $[0,0,0]^{T}$ is:

$$
\Sigma: \boldsymbol{n}^{T} \boldsymbol{p}=0
$$

Set up the equation about the sunlight. Supposing $\alpha_{2}$ is the solar altitude angle and $\beta_{2}$ is the azimuth angle, south by east about the azimuth angle is negative and south by west is positive. The solar altitude angle and azimuth angle can be calculated through the Eqs (1) and (2).

The sunlight's direction vector is:

$$
\boldsymbol{s}=R\left[z, \beta_{2}\right] R\left[y,-\alpha_{2}\right]\left[\begin{array}{lll}
1 & 0 & 0
\end{array}\right]^{T}
$$

Where, $R\left[z, \beta_{2}\right]$ and $R\left[y,-\alpha_{2}\right]$ is the spin matrix around $\mathrm{z}$-axis and $\mathrm{y}$-axis, then

$$
\begin{aligned}
& R\left[z, \beta_{2}\right]=\left[\begin{array}{ccc}
\cos \beta_{2} & -\sin \beta_{2} & 0 \\
\sin \beta_{2} & \cos \beta_{2} & 0 \\
0 & 0 & 1
\end{array}\right] \\
& R\left[y,-\alpha_{2}\right]=\left[\begin{array}{ccc}
\cos \alpha_{2} & 0 & \sin \left(-\alpha_{2}\right) \\
0 & 1 & 0 \\
-\sin \left(-\alpha_{2}\right) & 0 & \cos \left(-\alpha_{2}\right)
\end{array}\right]
\end{aligned}
$$


Substitute $R\left[z, \beta_{2}\right]$ and $R\left[y,-\alpha_{2}\right]$ into the Eq. (14), then

$$
\boldsymbol{s}=\left[\begin{array}{lll}
\cos \beta_{2} \cos \alpha_{2} & \sin \beta_{2} \cos \alpha_{2} & \sin \alpha_{2}
\end{array}\right]^{T}
$$

Supposing $H$ is the height between the photovoltaic arrays topside and the ground, the photovoltaic arrays topside intersecting with z-axis and $\boldsymbol{h}=[0,0, H]^{T}$ is an intersection point. The sunlight linear equation $\mathrm{L}$ through the point $\boldsymbol{h}$ is:

$$
L: \boldsymbol{p}=s t+\boldsymbol{h}
$$

Combining the ground Eq. (13) and sunlight linear Eq. (15), the intersection point $\boldsymbol{P}_{0}$ of the sunlight and sloping ground through the point $\boldsymbol{h}$ is:

$$
\boldsymbol{P}_{0}=\left[\begin{array}{lll}
x_{0} & y_{0} & z_{0}
\end{array}\right]^{T}=\frac{-\boldsymbol{s} \boldsymbol{n}^{T} \boldsymbol{h}}{\boldsymbol{n}^{T} \boldsymbol{s}}+\boldsymbol{h}
$$

Supposing the straight line $L_{2}$ is the projection of the sunlight line $L$ on the sloping ground. $\boldsymbol{s}_{2}=$ $p /\|p\|$ is the projection direction vector and passing through the origin point. So equation of the line $L_{2}$ is:

$$
L_{2}: \boldsymbol{p}=s_{2} t
$$

Supposing $\alpha_{4}$ is dip angle of the photovoltaic arrays sloping ground, and $\beta_{4}$ is the azimuth angle, south by east is negative and south by west is positive. When installed, the angle $\alpha_{3}$ of the hypotenuse with horizontal ground is generally adopted as the dip angle and equal to $\alpha_{4}$ if the ground is horizontal. The photovoltaic arrays azimuth angle is the azimuth angle $\beta_{3}$ of the topside's downward vertical plane, the south by east is negative and south by west is positive and equal to $\beta_{4}$ if the ground is horizontal.

Normal of the photovoltaic arrays topside's downward vertical plane is:

$$
\boldsymbol{n}_{2}=R\left[z, \beta_{3}\right]\left[\begin{array}{lll}
1 & 0 & 0
\end{array}\right]^{T}
$$

Where, $R\left[z, \beta_{3}\right]$ is the spin matrix around $\mathrm{z}$-axis, then

$$
R\left[z, \beta_{3}\right]=\left[\begin{array}{ccc}
\cos \beta_{3} & -\sin \beta_{3} & 0 \\
\sin \beta_{3} & \cos \beta_{3} & 0 \\
0 & 0 & 1
\end{array}\right]
$$

Substitute $R\left[z, \beta_{3}\right]$ into the Eq. (18), then

$$
\boldsymbol{n}_{2}=\left[\begin{array}{lll}
\cos \beta_{3} & \sin \beta_{3} & 0
\end{array}\right]^{T}
$$

Equation of the photovoltaic arrays topside's downward vertical plane is:

$$
\Sigma_{2}: \boldsymbol{n}_{2}^{T} \boldsymbol{p}=0
$$

The distance from the intersection point $\boldsymbol{P}_{0}$ of the sunlight with the ground to the vertical plane $\sum_{2}$ is also the distance between the photovoltaic arrays from the front to back. It can be calculated from distance formula of the point to plane.

$$
D_{1 h}=\frac{\left|\boldsymbol{n}_{2}^{T} \boldsymbol{P}_{0}\right|}{\left\|\boldsymbol{n}_{2}\right\|}=\left|\boldsymbol{n}_{2}^{T} \boldsymbol{P}_{0}\right|
$$


Horizontal projection $D_{1 h}$ of the shadow in the vertical plane $\sum_{2}$ is:

$$
D_{1 h}=\sqrt{x_{0}^{2}+y_{0}^{2}-\left|\boldsymbol{n}_{2}^{T} \boldsymbol{P}_{0}\right|^{2}}
$$

Combining the Eqs (19) and (13), intersection line of the photovoltaic arrays topside and the ground will be obtained. The direction vector $\mathbf{s}_{3}$ of the intersection line is:

$$
s_{3}=\boldsymbol{n} \times \boldsymbol{n}_{2}
$$

Equation of the intersection line going through the origin point can be obtained:

$$
L_{3}: \boldsymbol{p}=s_{3} t
$$

The distance $D_{1}$ from the intersection point $\boldsymbol{P}_{0}$ of the sunlight and the ground to the straight line $L_{3}$ is the photovoltaic arrays' minimum interval from the front to back on sloping ground.

$$
D_{1}=\frac{\left\|s_{3} \times\left(\boldsymbol{P}_{0}-\mathbf{0}\right)\right\|}{\left\|s_{3}\right\|}=\frac{\left\|s_{3} \times \boldsymbol{P}_{0}\right\|}{\left\|s_{3}\right\|}
$$

The projection $D_{2}$ on the straight line $L_{3}$ of the photovoltaic arrays' shadow on sloping ground is:

$$
D_{2}=\sqrt{\left\|\boldsymbol{P}_{0}\right\|^{2}-D_{1}^{2}}
$$

The height $H$ between the photovoltaic arrays topside and ground is directly adopted for the distance calculation. In general, for installing easily, it only gives azimuth angle $\beta_{3}$ of the photovoltaic arrays topside's downward vertical plane, and the photovoltaic arrays dip angle $\alpha_{3}$ of the hypotenuse and the horizontal ground, and the geometry parameters of the photovoltaic arrays. Calculation of the height $H$ needs the length of the hypotenuse $l$.

Supposing the vertex coordinate of the hypotenuse is $\boldsymbol{P}_{3}=\left[x_{3}, y_{3}, z_{3}\right]^{T}$, the vertex coordinate $\boldsymbol{P}_{3}$ can be calculated by solving the equations below.

$$
\left\{\begin{array}{l}
z_{3}=l \sin \alpha_{3} \\
\left\|\boldsymbol{P}_{3}\right\|=l \\
\boldsymbol{P}_{3} \cdot \boldsymbol{s}_{3}=0
\end{array}\right.
$$

The z-axis coordinate of the vertex $\boldsymbol{P}_{3}$ vertical downward projection on the ground is obtained by substituting $\mathrm{x}_{3}$ and $\mathrm{y}_{3}$ in the point $\boldsymbol{P}_{3}$ into the ground Eq. (13). It is represented by $z_{4}$. Consequently, the vertical distance $H$ between the vertex and the ground is:

$$
H=z_{3}-z_{4}
$$

\section{The actual calculation example}

The photovoltaic power station is built on the sloping ground of the dam. The dam's orientation is not the south and the shore is divided into four areas: the north, the south, the east and the west. The dip angle and azimuth angle of the sloping ground and photovoltaic arrays in each area are different, and more detailed situations are shown in Table 1 . The photovoltaic arrays are all along with the sloping ground in 
Table 1

Actual calculation example of the minimum distance from the front to back on the sloping ground

\begin{tabular}{lccccc}
\hline Ground position & $\begin{array}{c}\text { Ground } \\
\text { dip } \\
\text { angle }\end{array}$ & $\begin{array}{c}\text { Ground } \\
\text { azimuth } \\
\text { angle }\end{array}$ & $\begin{array}{c}\text { Photovoltaic } \\
\text { arrays } \\
\text { dip angle }\end{array}$ & $\begin{array}{c}\text { Photovoltaic } \\
\text { arrays } \\
\text { azimuth angle }\end{array}$ & $\begin{array}{c}\text { The minimum distance of } \\
\text { photovoltaic arrays from front } \\
\text { to back on sloping ground }(m)\end{array}$ \\
\hline North shore & -7 & 16 & 31 & 16 & 9.1 \\
South shore & -8 & 16 & 31 & 16 & 10.2 \\
& -9 & 16 & 31 & 16 & 11.4 \\
East shore & 7 & 8 & 31 & 8 & 2.7 \\
& 8 & 8 & 31 & 8 & 2.6 \\
West shore & 9 & 8 & 31 & 8 & 5.4 \\
& 8 & -90 & 31 & 0 & 5.8 \\
& 9 & -90 & 31 & 0 & 6.1 \\
& 7 & 106 & 31 & 0 & 4.3 \\
& 8 & 106 & 31 & 16 & 5.6 \\
\hline
\end{tabular}

Table 2

Calculation of the minimum distance between two photovoltaic arrays in particular cases

\begin{tabular}{lr}
\hline Case & Equation \\
\hline Arrays arranged in south direction on horizontal ground & $(4)(5)$ \\
Arrays arranged in south direction on north-south sloping ground & $(4)(5)(5)$ \\
Arrays arranged in south direction on east-west sloping ground & $(4)$ \\
Arrays arranged along with the sloping direction on arbitrary direction & $(4)(5)(10)$ \\
\hline
\end{tabular}

four areas, i.e., the azimuth angle of the dam sloping ground and azimuth angle of the photovoltaic arrays is identical. In this way, the calculation of the minimum distance about the photovoltaic arrays on sloping ground has two methods: one is considering the azimuth angle and calculating from the Eqs (4) to (7); the other is calculated in the Eqs (24) and (25). Length of the solar photovoltaic arrays' hypotenuse is $3.3 \mathrm{~m}$ and the optimal dip angle is $31^{\circ}$ (considering the angle of the hypotenuse and horizontal plane in the calculation). Firstly, Calculate the height $H$ in the Eqs (26) and (27). Then the minimum distance between the front and back on the sloping ground can be calculated in the Eqs (12), (14), (16), (18), (22), (24). Table 1 shows the calculation results. In Table 1 the positive dip angle indicates the condition that the south direction of sloping ground is low while the north is high. The negative indicates the opposite condition. The positive azimuth angle indicates the south by east direction and negative shows the south by west direction.

\section{Conclusions}

This paper introduces the calculation method on different ground conditions that the solar photovoltaic arrays' distance from the front to back, including horizontal ground, sloping ground in north-south direction, east-west direction and the calculation methods of the distance between the photovoltaic arrays on general sloping ground. It is summarized as follows.

\subsection{Calculation of the minimum distance between two photovoltaic arrays in particular cases}

The calculation method of the minimum distance between two photovoltaic arrays in particular cases is shown in Table 2. 


\subsection{Calculation of the minimum distance between two photovoltaic arrays in general cases}

The calculation procedure of the distance between two solar photovoltaic arrays on general sloping ground is shown as follows:

1) Confirm the height $H$ between the photovoltaic arrays topside and ground. $H$ can be calculated in the Eqs (26) and (27) when giving the photovoltaic arrays azimuth angle $\beta_{3}$, dip angle $\alpha_{3}$ and length of the hypotenusel.

2) Calculate the photovoltaic arrays downward vertical plane normal $\boldsymbol{n}_{2}$ through the azimuth angle $\beta_{3}$ and dip angle $\alpha_{3}$.

$$
\boldsymbol{n}_{2}=\left[\begin{array}{lll}
\cos \beta_{3} & \sin \beta_{3} & 0
\end{array}\right]^{T}
$$

3) Calculate the ground normal $\boldsymbol{n}$ through the normal's azimuth angle $\beta_{1}$ and altitude angle $\alpha_{1}$.

$$
\boldsymbol{n}=\left[\begin{array}{lll}
\cos \beta_{1} \sin \alpha_{1} & \sin \beta_{1} \sin \alpha_{1} & \cos \alpha_{1}
\end{array}\right]^{T}
$$

4) Calculate the sunlight direction vector $s$ through the sunlight azimuth angle $\beta_{2}$ and altitude angle $\alpha_{2}$.

$$
\boldsymbol{s}=\left[\begin{array}{lll}
\cos \beta_{2} \cos \alpha_{2} & \sin \beta_{2} \cos \alpha_{2} & \sin \alpha_{2}
\end{array}\right]^{T}
$$

Where, $\alpha_{2}$ and $\beta_{2}$ can be calculated in the Eqs (1) and (2).

5) Calculate the intersection point $\boldsymbol{P}_{0}$ of the sunlight and the sloping ground.

$$
\boldsymbol{P}_{0}=\frac{-\boldsymbol{s} \boldsymbol{n}^{T} \boldsymbol{h}}{\boldsymbol{n}^{T} \boldsymbol{s}}+\boldsymbol{h}
$$

Where, $\boldsymbol{h}=[0,0, H]^{T}$.

6) The minimum distance of the photovoltaic arrays from the front to back is:

$$
\frac{\left\|\boldsymbol{n} \times \boldsymbol{n}_{2} \times \boldsymbol{P}_{0}\right\|}{\left\|\boldsymbol{n} \times \boldsymbol{n}_{2}\right\|}
$$

\section{Acknowledgement}

This paper is supported by the Projects of Ningbo Innovation Team (2012B82002, 2013B82005).

\section{References}

[1] D. Weinstock and J. Appelbaum, Shadow variation on photovoltaic collectors in a solar field[C]. Electrical and Electronics Engineers in Israel, Proceedings of 23rd IEEE Convention of IEEE, 2004, pp. 354-357.

[2] Q.W. Wang, D.L. Yu, R.J. Shang et al., Numerical simulation of the influence of PV module spacing on daily generating output per unit area[J], Renewable Energy Resources 31(9) (2013), 13-17.

[3] Y.Z. Wu and L.J. Zou, The calculation of spacing between solar cell array in photovoltaic power station[J], Energy Engineering 30(1) (2011), 39-40.

[4] C.Y. Zhou, Z.Y. Yang and S.M. Yang, Design of array spacing for photovoltaic power station on northern slope area[J], Huadian Technology 35(6) (2013), 14-17.

[5] D. Díaz, Eloy, et al. Optimal distribution for photovoltaic solar trackers to minimize power losses caused by shadows[J], Renewable Energy 36(6) (2011), 1826-1835.

[6] C.G. Wang and S.C. Wang, Solar photovoltaic power generation technology[M]. Beijing: Chemical industry press, 2009.

[7] J. Hofierka and M. Súri, The solar radiation model for open source GIS: Implementatino and applications[J]. Trento, Italy: Chez Open source GIS - GRASS users conference, 2002, (9): 11-13.

[8] J. Duffie and W. Beckman, Solar engineering of thermal processes[J]. USA: John Wiley \& Sons, 1991.

[9] China federation of power enterprises. Design specification of photovoltaic power station[M]. Beijing: China Planning Press, 2012.

[10] W.S. Qiu, Analytic geometry[M]. Beijing: Peking University Press, 2013. 